separator. Then plaster is poured into the mold to produce a cast of the partial pot. Caution should be observed to make the cast as nearly the thickness of the pottery as possible, and to facilitate the easy removal of the mold.

While the cast is drying, the operator may continue his work by finding the circumference of the mouth of the original pot. The outside of the rim is traced on a piece of paper upon which the pottery section is resting, rim down. A flat pencil is used to permit the line to follow the rim more closely. With this done, the pottery is removed from the arc thus projected on the paper. On the arc draw two chords, and upon these chords erect perpendicular bisectors. The radius of the original vessel is the distance from the point of intersection of the bisectors to the arc.

The pottery piece is now fixed in erect position, resting on its rim, by driving wire brads to form inner and outer supporting rows. The cast reproduction of the pottery piece is placed on its rim, the latter resting on the line marking the completed circumference, opposite the true sherd. The next step is to model in the missing parts of the rim. This can be done in some cases by the application of thick plaster only. It may be necessary to model in clay the remaining spaces between the edges of the two pieces, and then build on either side a plaster mold which will overlap the edges of the pottery and the cast. The modeling clay will reconstruct the shape of the missing pottery, as indicated by the existing parts. Remove the clay after the plaster has hardened. Arrange the mold in a position so that the two pieces will be in their correct relative positions in the new mold, and thus add a cast that will make one solid piece of the original two. In many cases the missing parts of the vessel must be modeled in clay, and molds made, as in previously described instances. If the spaces to be filled are small, this can be effected from the outside by backing the opening with any kind of modeling material, filling in plaster, and finishing before the plaster is too hard.

Fred Carder

Norman, Oklahoma

\title{
Standardized TERMinology
}

Dr. Ray's comments on greater accuracy in terminology, offered in the January issue of AmERican Antiquity, are much to the point. I believe such a scheme as that which he outlines is at present being mulled over by various men in the field. Among others, Mr. M. R. Harrington of the Southwest Museum, and, unless I am misinformed, Dr. Arthur C. Parker of the Rochester Municipal Museum have been gathering data bearing on this problem. There have been several sporadic attempts in this direction, but few that have borne fruit.

There is a tendency among students new to the field to parrot expressions gleaned from previous publications without studying them. Moreover, they do not study specimen collections from the various archaeological areas, and 
photographs, sketches or wash drawings of specimens are likely to be deceptive.

Then, too, each area has a certain number of colloquialisms that have crept into the professional patter, and these are perpetuated in "sacred" print, and so become accepted.

It seems high time to call a halt on the broad use of terms which have a limited value, or at best a purely local application. Thus, in the Southwest we have the word olla; I have no objection to the word as applied to a specific type of earthen jar, but to apply it indescriminately to all manner of earthen vessels, and at times to those made of stone, seems a bit unreasonable. Other words, such as metate and mortar, or mano and pestle, are confused by collectors and amateur archaeologists. Collectors use the word arrowhead without understanding the functions of an arrow. Some use a most arbitrary method of determining arrowheads and spearheads, such as length. I have heard some collectors state that anything under two inches in length was an arrowhead, forgetting that bone arrowheads of the Eskimo may range up to ten or eleven inches in length; and the split-cane arrowheads from South America, even longer. Some of the bone arrowheads from the Plains are three or four inches in length, and a delicately fashioned stone arrowhead might well be over two inches in length, depending upon the length and strength of the bow in the particular area, the diameter and length of the arrow used, and the ability of the stone workers.

Going a bit further than Dr. Ray suggests, I should like to see a revision of terms; in other words, the compiling of an archaeological dictionary involving the discarding of those terms now in use which are not accurate or generally applicable. Mr. Harrington and I began such a classification. Some of the readers may not realize the difficulties involved in an attempt to define a stone axe, an arrow, or a bone awl in simple, easily understood language that could be accepted by the archaeologist in Maine, Alaska or Texas. True, there are many words now in use which could be retained, but the meanings would have to be clarified and standardized for all archaeologists. Pottery forms should be analyzed and a glossary of basic types drawn up. These could serve as common denominators. Each area would no doubt produce odd forms; in that case, the archaeologist would be free to add a local definition which would in no manner change the meaning of the basic form. In writing his report, he could preface it with his outline glossary, similar to that used by Drs. Kidder and Lothrop in The Pottery of Costa Rica and Nicaragua, Museum of the American Indian, Heye Foundation, Vol. I, pp. 109-110, New York, 1926. The dictionary, once compiled, might of a necessity undergo many revisions and editions as the science of archaeology progresses, but at least it would be a rock to which present and future archaeologists might anchor, and would bring about a greatly desired uniformity of language.

\author{
Arthur WoOdward \\ University of California \\ Berkeley, California
}

